\title{
Overview on intelligent comprehensive evaluation methods
}

\author{
Yong Yang ${ }^{1}$, Chenxia Suo ${ }^{2 *}$, Weijie $\mathrm{Hao}^{2}$, Zhihui Zhang ${ }^{2}$ \\ ${ }^{1}$ Postdoctoral Programme, Bank of Zhengzhou, Zhengzhou 450018, China \\ ${ }^{2}$ Beijing Institute of Petrochemical Technology, Beijing 102617, China
}

Corresponding Author Email: suochenxia@bipt.edu.cn

https://doi.org/10.18280/rces.050401

Received: 11 October 2017

Accepted: 26 September 2018

\section{Keywords:}

intelligentization, comprehensive evaluation, research overview

\begin{abstract}
As the computer technology develops, intelligent methods play an increasingly wider role in social life. Intelligent methods are self-adaption and self-organization oriented; exhibit very strong robustness and obvious merits in solving qualitative and quantitative problems, as well as confirming the qualitative and uncertain issues. This paper sorts out the important theories and methods for intelligent evaluation, analyzes and defines the basic principles and models involved, and forecasts the application of intelligent methods in comprehensive evaluation.
\end{abstract}

\section{INTRODUCTION}

Humans have not been very familiar with the mechanism of human brain to date, and even the academic circle does not have a unified cognition for the definition of "intelligence" either. Generally speaking, "intelligence" is a kind of measurement to the ability of solving problems, and a way to express a kind of thinking and experience. Those with the characteristics of "intelligence" can be regarded as an intelligent individual or intelligent system.

Firstly, the intelligence is an important feature of the intelligent systems. How to evaluate the intelligence of the intelligent systems is not only a very critical subject, but also a new field for artificial intelligence research. At present, the academic circle still does not have a unified cognition regarding the concept of the intelligence itself, and does not have an explicit definition either. As to how to define the intelligence, on one hand, factors including the environment, age, condition, etc. should be considered during evaluation, which should be relevant, relative and time-based. On the other hand, there is still a lack of special researches on the intelligence. Intelligent control systems and intelligent products are getting more and more popular, and many "intelligent" products and systems are available in the market. Therefore, the special study on intelligence evaluation is quite necessary from both academic and application perspectives.

Secondly, what is the "intelligence" is an important characteristic of intelligent comprehensive evaluation methods. According to Xu Xuyan and other scholars, the systems and products can be regarded as intelligent systems and products if they have multiple features at the thought layer, perception layer and behavior layer respectively. Liu Dong and other scholars [1] calculated and evaluated the level of intelligence. This paper believes that intelligence refers to intelligence that simulate, extend and expand life mechanism, brain intelligence and group intelligence. The qualitative evaluation of the intelligence of intelligent systems includes self-recognition, self-planning, self-learning, self-adaptation, self-optimization, self-organization, self-coordination, selfreproduction and self-restoration.
At last, what is "intelligentization"? Regarding intelligentization, Nilsson from the Stanford University proposed that artificial intelligence is about the science of knowledge, in particular, the representation, acquisition and application of the knowledge. Liu Dong and other scholars [2] put forward the generalized concept of intelligent evaluation; and also pointed out that intelligentization refers to theories, methods and technologies involving anthropopathic intelligence or using artificial intelligence. Intelligentization in this paper refers to a kind of method integration that the systems apply the theories or methods with artificial intelligence to some areas to make the systems intelligent.

Two research approaches of artificial intelligence are available in generally. The first one is to adopt computer science to simulate the functions of human brain to realize artificial intelligence; that is, to use the operation of computer programs to achieve the effects similar to intelligent behavior activity process of humans. The expert system, which is called the traditional artificial intelligence, is the most active and most widely applied artificial intelligence in current researches. The other approach is to research the intelligent activities of human brain from the perspective of physiology, so as to unveil the secret of human intelligence. The structure of human brain must be made clear by the approach, that is, to simulate the mechanism that human brain processes the information through the bionic research on the nerve cell mechanism of human brain, so as to realize the artificial intelligence function of machines. A typical example of the research approach is the research on the artificial neural network. However, at the current stage, it is still very difficult to precisely simulate human brain in physical experiment, and a lot of researches need to be conducted in the future, since human brain has tens of millions of nerve cells. Up to now, the above two approaches have been only basic ideas about artificial intelligence research. The two methods were almost put forward and researched at the same time; and each of them was developed with hard efforts, which not only showed the determination and perseverance of humans' in exploring the secret of intelligence, but also indicated the hardship of intelligence research [3]. 


\section{MEASUREMENT OF INTELLIGENTIZATION}

The intelligent systems are organizational system models with human-imitating intelligence [4]. The human-imitating intelligence refers to the simulation, extension and expansion of the human functions with intelligence, including the learning ability, adaptation ability, organization ability, search ability, recognition ability, stabilizing ability, coordinating ability, planning ability, reproductive ability and repair ability. Humans are multi-level and multi-system intelligent control systems; so the simulation of human functions can be divided into different levels and different systems according to the level of intelligence, such as, high-level intelligent systems, middle-level intelligent systems and low-level intelligent systems. The common simulation intelligent models include the artificial neural network, the expert system and the support vector machine. Tu Xuyan [4] pointed out if an intelligent system or product had multiple intelligences, including the thinking ability, self-behavior ability and sensory ability, the system can be called an intelligent system. Cai Zixing, et al. [5] proposed that if a system or product adopted multiple intelligent theories, methods and technologies, the system or product should be an intelligent system or product with multiple intelligences. Liu Dong [1] studied how to evaluate the intelligence level of the intelligent control systems, and put forward corresponding algorithm and method. This paper puts forward the intelligent evaluation model framework of the intelligent systems on the basis of qualitative evaluation of intelligence.

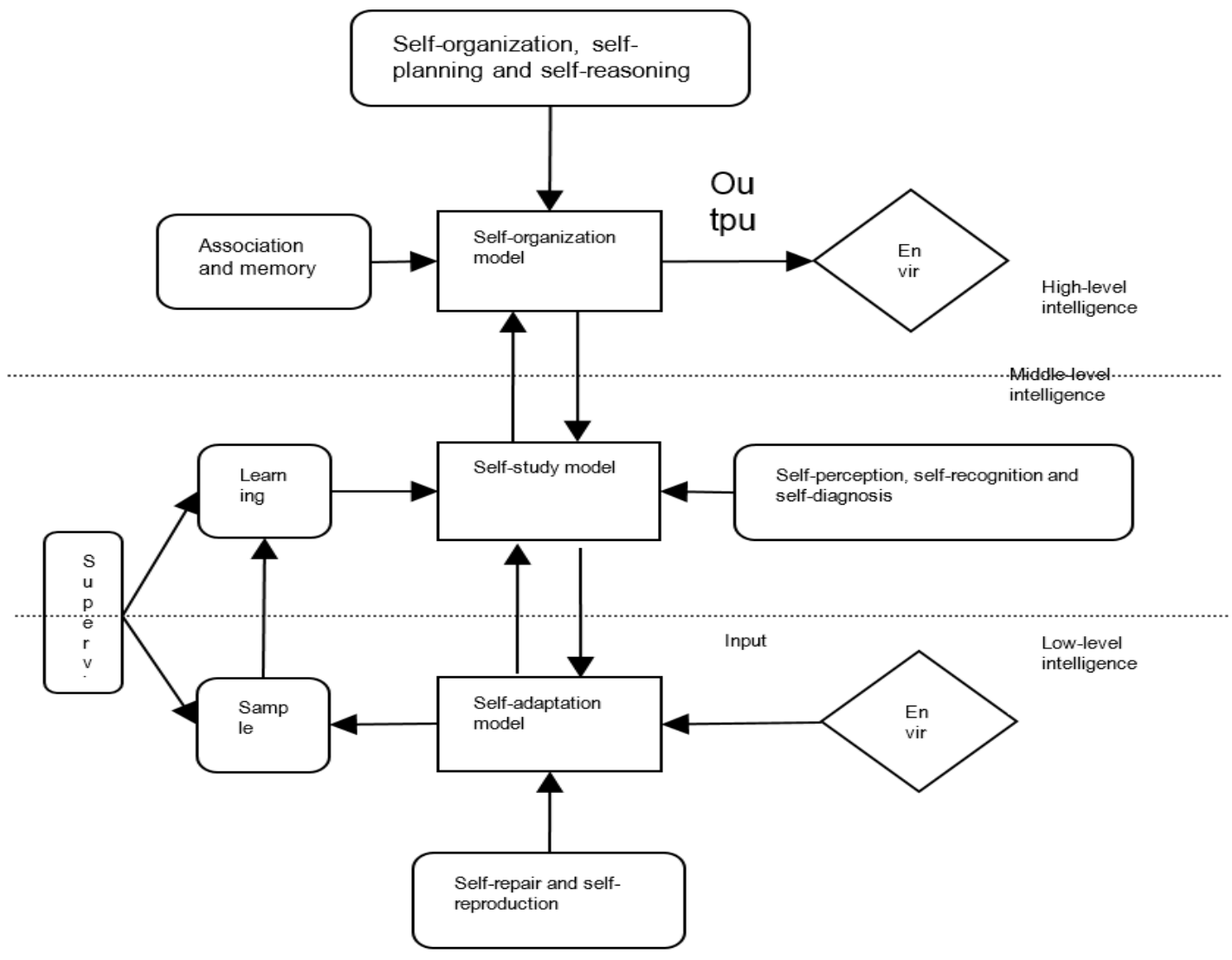

Figure 1. Intelligent model

It can be learnt from Figure 1 that the intelligent model itself is featured by network and circulation, and it is also expandable and modifiable; therefore, the model is a system with intelligence. The input of the system is the evaluation information provided by the external environment, which can be expert opinions, survey data, etc. The output is the reaction of the system against the external environment, which can be behaviors, data, modes, etc. that expand the functions of the intelligent system and promote the intelligent level of the intelligent system.

\section{MAIN INTELLIGENT EVALUATION METHODS}

\subsection{Expert evaluation system method}

The expert system refers to the intelligent system that relies on experts' experience and professional knowledge accumulated in specific fields to solve problems in similar fields through simulating experts' thinking model in solving problems.

The role of the expert system in comprehensive evaluation mainly includes two aspects: the extraction of expert knowledge, and the building of simulating expert evaluation model. In comprehensive evaluation, expert knowledge is often used to evaluate the evaluation object; and the comprehensive evaluation model based on the expert system is called the intelligent evaluation expert system (IEES) in this paper. The system uses the computer software system in a specific field and the reasoning techniques in artificial intelligence to understand and process professional complex problems that experts can solved. Its working principles are as follow: search expert experience and knowledge in specific fields, summarize and sort-out these experience and 
knowledge, find out the knowledge implied in expert judgment through analysis, judgment and reasoning, and use the computer system to realize and simulate the evaluation function of the experts. The system is mainly composed by knowledge base, comprehensive data base, inference subsystem, explanation subsystem, man-machine interface, knowledge acquisition and other functional modules, with the knowledge base and inference subsystem being the core parts. In view of these issues involved in evaluation, it needs to extract and simulate the fuzzy rule based on expert evaluation, transform evaluation standards to rules, store them in the knowledge base, and evaluate the object through transferring the expert knowledge in the knowledge base and rules base, as shown in Figure 2.

At present, the expert system has been successfully applied to many fields, including personal credit evaluation, risk evaluation, disaster diagnosis, etc. Grzymala Buser, et al. [6] put forward a type of LEMS algorithm of extracting minimal decision rules, which is widely applied to the classification and exploration of medical knowledge; Wang Zongjun, et al. [7] employed the expert system (CCEES) in the comprehensive evaluation of urban development level, so as to establish the basic structural pattern on the basis of artificial neural network (ANN) and expert system (ESS) integration method; they also introduced the evaluation index standardization method, the knowledge acquisition ANN method, the decision table knowledge base automatic generation method and the working principles of the inference machine in CCEES in details. Law and Au [8] studied the classification issues in numeric-type and non-numeric type blended data set and analyzed the shopping rule on tour through extracting the decision rules. Wang [9] used the BP neural network model to evaluate the risks in supply chain, and amended the weight of the model constantly during selflearning to make the actual output vector of network gradually approach the expected output value. Finally, he identified through weight matrix analysis that the expert system judgment supply chain risk evaluation model had no impact to the main risk factors in supply chain. Sun Xuejun [10] used the expert system to conduct early warning, assessment and evaluation of the safety status of coal mines.

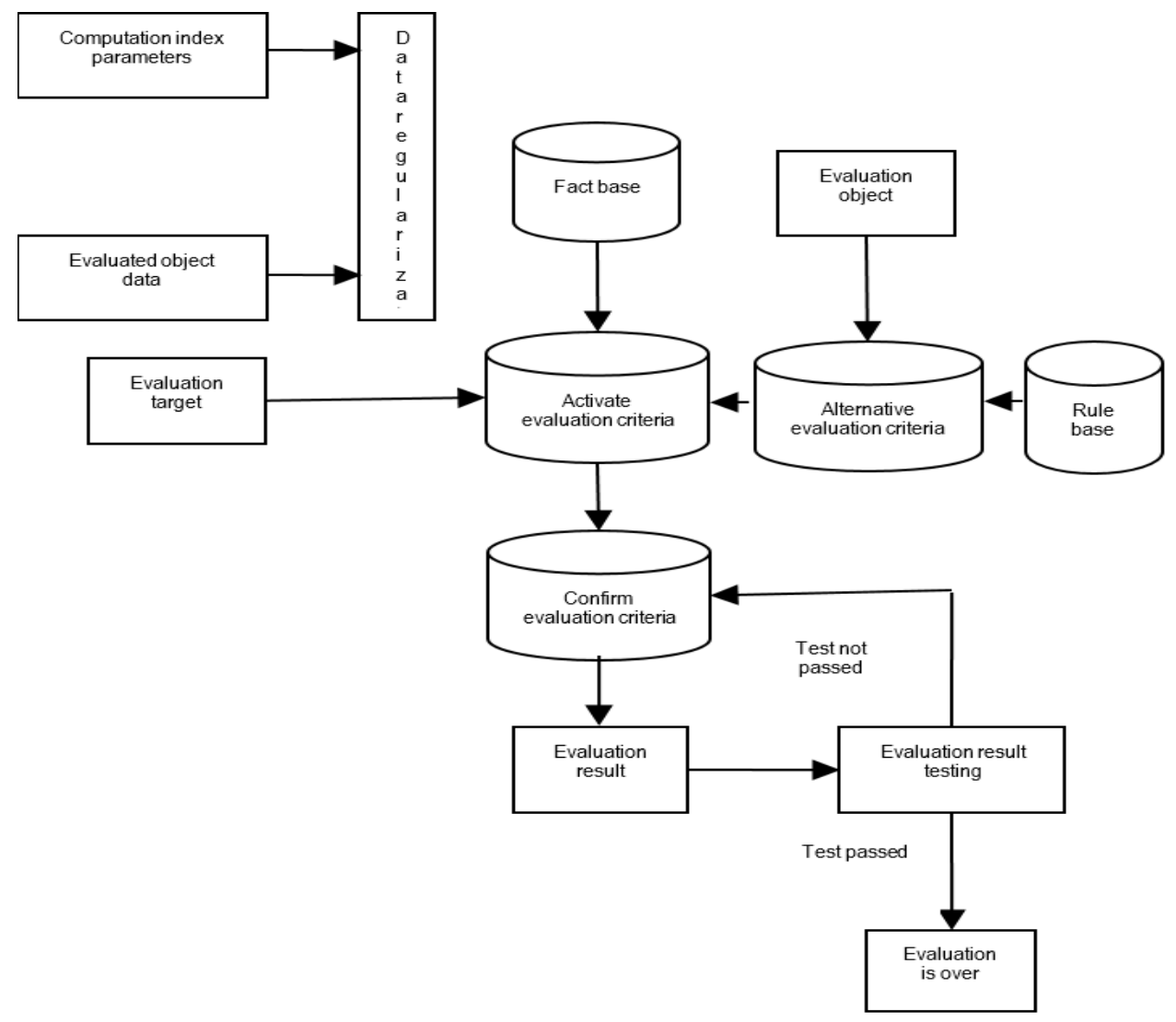

Figure 2. Comprehensive evaluation module based on expert system

\subsection{Machine learning evaluation method}

Machine learning (ML) refers to the acquisition of knowledge independently through machines. At present, ML's main contents include the learning mechanism of human brains, the thinking process of human brains, how to build the learning system model of specific tasks, and how to make the machines learn and acquire knowledge. Some research subjects in robotics are involved as well, such as the moving path planning of robots and the planning methods of robot target action sequence; with the research on how to transform advanced intelligent robots to modern robots being an important content.

The ML ability is not only an important approach for computers to acquire knowledge, but also a distinctive feature of artificial intelligence. It studies how to simulate or realize human learning function subjects with computers, as well as how to acquire new knowledge and skills through machine recognition with existing knowledge. It is generally accepted that the ML is a knowledge acquisition process with specific targets, whose mechanism is a knowledge accumulation process from unknown to known. Its system performance is 
about the improvement of performance and adaptation abilities, making the system realize functions that cannot be realized or complete tasks that cannot be finished before. This is the process of knowledge accumulation, as well as the improvement process of knowledge acquisition skills.

The ML is an active field in the artificial intelligence research, that studies how to make computers have the ability of acquiring knowledge in the real world like humans, build the computing theory of the ML according to the principle of simulating the learning process of humans, and develop various learning systems and make them play a role in actual application. Currently, researchers have been continuing deepening the research on the ML, and put forward multiple learning systems and methods under different research environment and in various fields. Therefore, the target of the ML is to make the machines have creative thinking, and make them be able to summarize, learn and find out knowledge independently from a great deal of knowledge input, so as to make a greater contribution to human development.

The ML has been applied to the comprehensive evaluation of web content quality evaluation, credit evaluation, image quality evaluation, etc. [11-12]. The evaluation objects should be classified before processing when the ML method is adopted, so as to avoid the difficulties lying in direct quantitative processing. Good evaluation effects can be obtained with the learning mechanism boasting plenty of training samples. The most important strategy in the ML is case learning, which is a learning method to conclude the generalized concept through extracting several relevant concepts from the environment.

The ML method is developed based on the statistical learning theories and nuclear techniques, and its core content is the statistical machine learning method with the supporting vector product being the core algorithm [13]. The ML method can be divided into the supervised learning method and nonsupervision learning method based on different learning styles. The former one, which was raised [6], mainly refers to the learning method based on supporting vector product with good mode recognition ability and classification ability. The reproducing kernel function is adopted by the support vector machine to realize the function of the non-linear support vector machine, and in turn enhance the learning ability of support vector machine. Burges, et al. [14] proposed the improvement methods for the computational accuracy, computation speed and applicability of the mode recognition support vector machine. Scholkopf [15], Campbell [16] and Lee Y.J. [17] analyzed and improved the computational accuracy of the regression estimation support vector machine, as well as the expression form and stability of loss function. Lee, et al. raised the method of the smooth support vector machine which was applied with good results in the mode recognition. Chen Wei, et al. [18] put forward a domain name credit evaluation automatic method based on the ML, which was proved to be better in line with people's general view through experimental analysis with more accurate results, so as to overcome the defects of the least square support vector machine, including high computational complexity and non-sparseness.

\subsection{Artificial neural network evaluation method}

The artificial neural network (ANN) is an artificial system that employs computer science and engineering technology methods to simulate the neural network structure and functional features of human brains. The ANN is a non-linear processing unit that simulates the neural units in human brains, matches synaptic behavior characteristics through alterable connection strength, and makes the network structure form a large-scale collateral non-linear power system. The artificial neural network shows parallel processing capability, selforganization ability and fault-tolerant ability during processing of large-scale data, therefore, the network model has very strong learning, memory, association and recognition abilities. Hopfield [19] put forward the HNN model which introduced the concept of energy function. Le Cun and Rumelhart raised the counter propagation algorithm of all nerve cell weights in the structure sensor, namely, the BP algorithm. The artificial neural network simulation method is an analogy procedure based on the intelligent structure with several merits. For example, it does not need to know the model structure in advance, but need to complete model prediction through training self-learning function. The artificial neural network has strong non-linear processing ability, which can not only overcome the defect of the traditional artificial intelligent method in speech recognition and model recognition; but also expand the application range of the model, becoming the major contribution to modern artificial intelligence theories.

The self-learning ability of the artificial neural network is realized through two processes: forward propagation process and counter propagation process. The former process means that after information enters into the layer of nerve cells, it is processed layer by layer until it is delivered to the nerve cells in output layer. The state of nerve cells in each layer is only influenced by of nerve cells in the last layer. When the nerve cells in output layer receive different results and preconception, the propagation process will be reversed. The role of counter propagation is to turn back the error information to the last layer along the original forward propagation path for amendment, reduce errors through amending the weight of the original nerve cells, and repeat the process back and forth until the error information received is less than expected ones. The connection among nerve cells shows the exchange of knowledge and information; while the learning and recognition process of network exhibits the adjustment process of the nerve cell connection weight.

Nowadays, the integration of the nerve neural network model and other intelligent methods are extensively applied in various aspects, including signal processing, model recognition, robots, expert systems and network safety.

\subsection{Monte Carlo simulation evaluation method}

The Monte Carlo method is similar to statistical methods and uses the occurrence frequency of the computer simulation random event as the estimated value to show the probability of any event [20]. It is a statistical simulation experiment of the computer; and a numerical simulation method of the random event actually, with its main steps being as follows: simulate the evenly distributed random number sequence $u_{i}$ in the section $[0,1]$, transform $u_{i}$ to the required distributional random variable $\mathrm{x}_{\mathrm{i}}$ by the way of function transformation, and then research according to the distribution of random variable $\mathrm{x}_{\mathrm{i}}$. The common transformation method is the reversed transformation method. Generally speaking, the core of the Monte Carlo simulation method includes two steps: firstly, simulate the even distribution of $[0,1]$ and then adopt function transformation to reach the required distribution. At present, the common method for simulating even random number 
sequence is the multiplicative congruence method, and its basic steps are as follows: first of all, select the multiplier A, divisor $\mathrm{M}$ and initial value $\mathrm{u}$; then use the recursion formula $\mathrm{u}_{\mathrm{i}}=\bmod \left(\mathrm{Au}_{\mathrm{i}-1}, \mathrm{M}\right), \mathrm{i}=1,2, \ldots, \mathrm{n}$, to establish the random sequence; mod is the complementary function, $\bmod \left(\mathrm{Au}_{\mathrm{i}-1}, \mathrm{M}\right)$ is divided by $\mathrm{Au}_{\mathrm{i}-1}$ to get the remainder $\mathrm{u}_{\mathrm{i}}$, and then transform $\mathrm{u}_{\mathrm{i}}$ to even random number sequence $\mathrm{x}_{\mathrm{i}}$ in the section $[0,1]$ through function transformation $x_{i}=u_{i} / M$. It is important to note that the random distribution type of the research object should be confirmed according to empirical assumption or historical data when using the Monte Carlo method.

In comprehensive evaluation, the Monte Carlo simulation method is required to be adopted for many random problems so as to obtain required data through simulation test, and then obtain relatively quantitative results. The simulation method is mainly applied in the following aspects in intelligent comprehensive evaluation: risk evaluation, decision optimum selection, original state setting, simulation model generation and policy simulation. For instance, in the test of the evaluation model, the simulation data may be used to test the effectiveness of the evaluation model [21]; the random simulation method may be resorted to process subjective evaluation problems with high index weight; and the simulation method may be used to confirm the distribution of uncertain factors, such as, the distribution of objective evaluation information and the subjective evaluation reliability.

\subsection{Genetic algorithm evaluation method}

Genetic algorithm is a new optimization method combining the certainty optimization method and the complete random optimization method. The certainty method is expressed in the application of the fundamental of biological evolution law of "survival of the fittest", and the selection operation of individual "inheritance" through the height of fitness function for the next round of iterative evolution process. While the random method is mainly showed in random selection, hybridization and mutation operations, which are aimed at stimulating the random feature of genetic inheritance, mutation and variation in the biological evolution process. In brief, the genetic algorithm, as an optimization method, is realized through simulating the intelligent process; and it is better than conventional optimizing method because it directly faces the optimization issue. The optimized results are not about a single solution, but a set of optimal solutions. By this way, there's an opportunity to choose solutions, and there is much more flexibility in processing complex system evaluation problems. Specifically, the parent of genetic algorithm is to code the feasible parent solutions (alphabetic string) through the random formulation of feasible solutions, with the fitness function of the parent acclimatization ability being the optimization target (a transformation of objective function); and generate offspring individuals through constant selection, hybridization and mutation operations, with the height of fitness being the selection criteria according to the rule of survival of the fittest, until the appearance of qualified off springs through the repetition iterative operation and the termination of iterative process In a sense, the intelligent optimization process of genetic algorithm is a process to optimize self-adaptation through simulating mass evolution, so as to constantly approach the optimal point under the influence of the individuals and environment. The individuals used in coding indicate the evolutionary direction is the optimizing direction of objective function under the constraint of environment.

In comprehensive evaluation, the genetic algorithm is often combined with other method models to form an intelligent comprehensive evaluation method. Taking the combination of the genetic algorithm and neural network as an example, the model uses the global searching ability of the genetic algorithm to optimize the initial weight of neural network [22]; and employs the evolution thought of genetic algorithm to process group decisions, in the combination of the genetic algorithm and GDSS evaluation model based on the decisionmaking performance level of the value evaluation group regarding genetic operators. The model also uses the algorithm optimization ability to optimize the function of its projection direction by taking into account the genetic algorithm and projection pursuit cluster evaluation model, transforms multidimensional data space to low-dimensional subspaces [23]; and makes use of the non-linear optimizing ability of the genetic algorithm in multi-objective planning to solve the problems regarding multi-objective decisions [24].

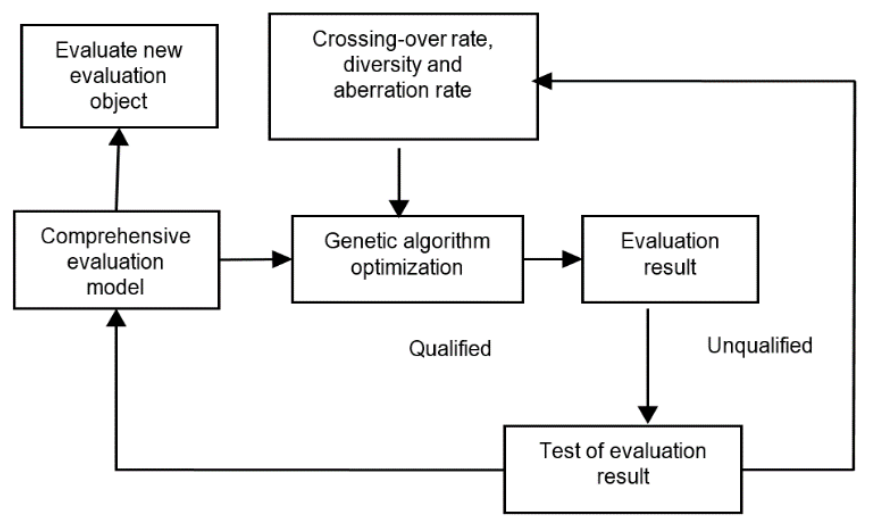

Figure 3. Combination of genetic algorithm and other evaluation models

Generally speaking, the genetic algorithm, as a global optimization method, plays a significant role in comprehensive intelligent evaluation, and solves many problems which cannot be solved with traditional evaluation models together with other evaluation models combined. The combining process is shown in Figure 3.

\section{OUTLOOK OF INTELLIGENT EVALUATION METHOD}

The development of the intelligent methods has opened up the new era of mathematics-based evaluation methods, dramatically enhancing the adaptability of evaluation methods. The comprehensive intelligent evaluation is a new research field; and is widely applied since its appearance; especially in the fields that traditional evaluation methods are not competent. The effectiveness of the intelligent methods is also fully proved in fields including combination evaluation, multiobjective and multi-stage evaluation, and complex system evaluation. The Introduction of intelligent methods in comprehensive evaluation has improved both the mathematical foundation and the intelligence of comprehensive evaluation, enriched the method system of evaluation, and provided strong methodological basis for problems in traditional evaluation, such as, poor information 
and gray information systems, evaluation in the uncertain state, and dynamic system evaluation. The comprehensive intelligent evaluation method is widely applied in the practical application because it is simple and effective; particularly in solving evaluation problems with complex structure; and it has the incomparable merits that traditional evaluation methods do not have; therefore, the comprehensive intelligent evaluation has attracted much attention from researchers. It can be seen from the research literatures in recent years, the application scope of comprehensive intelligent evaluation is getting wider and wider. The research on comprehensive intelligent evaluation keeps moving forward with further research on algorithm application. On the one hand, brand new progress has been achieved in the research of artificial intelligence theories; on the other hand, the computer technologies also realize leapfrog development. Many job tasks that cannot be completed before have been executed by now thanks to the continuous improvement of computer operation speed and the constant development of cloud intelligence and big data technology, which will promote the application of the intelligent methods in comprehensive evaluation.

\section{ACKNOWLEDGMENT}

This paper was funded by three projects: BIPT-POPME; Development Research Centre of Beijing New Modern Industrial Area (2016); BIPT-ER(2014); URT2017J00120.

\section{REFERENCES}

[1] Liu D, Yin YX, Tu XY, Dong J. (2005). An evaluation method on intelligent control system intelligent level. Journal of Central South University Special 13-16.

[2] Liu D, Yin YX, Tu XY. (2007). Research on generalized intelligent qualitative evaluation of intelligent system. Computer Science 34(9): 167-169.

[3] Huang W, Nie D, Chen YJ. (2001). The main school and characteristics in AI research. Journal of Gannan Teachers College (3): 73-75.

[4] Tu XY. (1994). Theories, methods and techniques of intelligent control, second national intelligent control expert seminar collected papers (1). Tsinghua University: 27-34.

[5] Cai ZX, Xu G. (2005). Artificial intelligence control. Beijing: Chemical Industry Press 3-20.

[6] Boser BE, Guyon IM, Vapnik VN. (1992). A training algorithm for optimal margin classifiers. Proceedings of the 5th Annual ACM Workshop on Computational Learning Theory 5: 144-152. https://doi.org/10.1145/130385.130401

[7] Wang ZJ. (1998). Methods, problems and research trends of comprehensive evaluation. Journal of Management Sciences in China 1(1): 73-79.

[8] Law R., Au N. (2000). Relationship modeling in tourism shopping: a decision rules induction approach. Tourism
Management 21(3):

https://doi.org/10.1016/S0261-5177(99)00056-4

241-249.

[9] Wang Q, Wang XL. (2005). Research on text classification techniques integrated $\mathrm{KNN}$ and SVM. Chinese High Technology Letters 15(5): 19-24.

[10] Sun XJ. (2011). Research on coal mine safety expert index evaluation system. Coal Economic Research (3).

[11] Jing HF, Wang B, Yang YH, Xu Y. (2009). Category distribution-based feature selection framework. Journal of computer research and development 46(9): 1586-1593.

[12] Yang Y, Slattery S, Ghani R. (2002). A study of approaches to hypertext categorization. Journal of Intelligent Information Systems 18(2): 219-241. https://doi.org/10.1023/A:1013685612819

[13] Vapnil VN. (1999). An overview of statistical learning theory. IEEE Transactions on Neural Networks 10(5): 988-999. https://doi.org/10.1109/72.788640

[14] Burges CJC. (1999). Geometry and invariance in kernel based method. Advances in Kernel Methods-Support Vector Learning, Cambridge: MIT Press 89-116.

[15] Scholkopf B, Simard P, Smola A, Vapnik V. (2000). Prior knowledge in support vector kernels. Advances in Neural Information Processing Systems (12): 526-532.

[16] Campbell C, Cristianini N, Smola AJ. (2000). Query learning with large margin classifiers. Proceedings of the 7th ICML, Stanford, pp. 111-118.

[17] Lee YJ, Mangasarian OL. (2001). SSVM: A smooth support vector machine for classification. Computational Optimization and Applications 20(1): 5-22. https://doi.org/10.1023/A:1011215321374

[18] Chen W, Wang L, Geng G, Mao W, Li X. (2012). Domain name credit evaluation method based on machine learning. Computer Application Research 29(2): 690-692.

[19] Hopfield JJ. (1984). Neurons with graded response have collective computational properties like those of twostate neurons. Proceedings of the National Academy of Sciences of the United States of America 81(10): 3088. https://doi.org/10.1073/pnas.81.10.3088

[20] Guo ZW, Xu LC, Zhu LQ, Cao YH. (1992). Theories and methods of macroscopic quality evaluation, enterprise development and system engineering. Beijing: China Science and Technology Press: 147-150.

[21] Liao YL. (2010). Comparison of two CSI computing methods based on customer evaluation changes. Mathematical Statistics and Management 29(4): 743-753.

[22] Zhu XD, Feng TJ. (2003). Personal credit evaluation based on GA neural network. System Engineering Theories and Practice 23(3): 48-51.

[23] Huang YH., Zhu, JF. (2009). Research and application on projection pursuit cluster evaluation model based on accelerating genetic algorithm. System Engineering 27(11): 107-110.

[24] Ni SY, Pan D, Wu CF. (2003). Comprehensive evaluation research on fund performance based on genetic algorithm. System Engineering 21(2): 1-6. 